\title{
Newly diagnosed asthma in China: initial severity and changes over a 1-year management period
}

\author{
Zhizhen $\mathrm{Hu}^{1}$, Jianwei Xuan ${ }^{2}$, Haijin Zhao ${ }^{1}$, Hangming Dong ${ }^{1}$, Changhui Yu ${ }^{1}$, Shaoxi Cai ${ }^{1}$, Yue Gao ${ }^{3}$, \\ Liran $\mathrm{Li}^{3}$, Xiaohan $\mathrm{Hu}^{3}$ \\ ${ }^{1}$ Department of Respiratory and Critical Care Medicine, Chronic Airways Diseases Laboratory, Nanfang Hospital, Southern Medical University, \\ Guangzhou, China; ${ }^{2}$ Health Economic Research Institute, Sun Yat-sen University, Guangzhou, China; ${ }^{3}$ Centennial Scientific Co., Ltd., Shanghai, \\ China \\ Contributions: (I) Conception and design: Z Hu, S Cai, J Xuan, Y Gao; (II) Administrative support: None; (III) Provision of study materials or \\ patients: None; (IV) Collection and assembly of data: Z Hu, S Cai, J Xuan, Y Gao; (V) Data analysis and interpretation: Z Hu, S Cai, J Xuan, Y Gao, \\ X Hu, H Zhao, H Dong, C Yu; (VI) Manuscript writing: All authors; (VII) Final approval of manuscript: All authors. \\ Correspondence to: Shaoxi Cai. Department of Respiratory and Critical Care Medicine, Chronic Airways Diseases Laboratory, Nanfang Hospital, \\ Southern Medical University, Guangzhou, China. Email: hxkc@smu.edu.cn.
}

Background: There are no large sample, epidemiological data describing initial asthma severity and change. We used a large health care database to examine asthma severity at initial diagnosis, and the changes in severity over the first year of management.

Methods: The clinical data of patients diagnosed with asthma for the first time were collated from the SuValue electronic medical database. The following inclusion criteria were applied: (I) patients who were 14 years or older at the time of first diagnosis; (II) initial diagnosis occurred between Jan 2001 and Mar 2019; (III) patients were followed up for at least 12 months; (IV) patients had follow-up visits every 3 months. Disease severity at diagnosis and at each follow-up visit, medications prescribed were collated and analyzed.

Results: A total of 7,654 adult patients with newly diagnosed asthma from tertiary hospitals (26.38\%) and secondary hospitals (73.62\%), who were followed up for at least 12 months, were included in this retrospective analysis. Approximately 54\% of patients were females and the largest age group was over 60 years old (37.66\%). Nearly $16 \%$ of patients were moderate to severe asthma initially. The proportions of patients with moderate and severe asthma decreased during the first 6 months, and remained stable thereafter. At the end of the 1-year follow-up period, $2.7 \%$ of patients had severe asthma. Patients with mild asthma tended to continue to have mild asthma in the following 3 months $(>76.19 \%)$. However, of the patients with mild or moderate asthma at 3 months, $92.85 \%$ and $75.1 \%$, respectively, experienced maintenance and reduction in severity and had mild asthma by 12 months. $1.26 \%$ and $3.15 \%$ of patients with mild or moderate asthma, respectively, progressed to severe asthma by 12 months.

Conclusions: Patients with mild asthma did not progress but rather, remained stable with mild asthma over the year. A proportion of patients diagnosed with moderate and severe asthma remained stable over a 1-year period. Further studies should be conducted to examine the clinical features of newly diagnosed patients with severe asthma without reduction in severity in order to facilitate intensive treatment and reduce the disease burden for these patients.

Keywords: Asthma severity; new diagnosis; China; treatment

Submitted Nov 12, 2021. Accepted for publication Jan 11, 2022.

doi: 10.21037/atm-21-6470

View this article at: https://dx.doi.org/10.21037/atm-21-6470 


\section{Introduction}

Bronchial asthma is a common chronic respiratory disease, and its prevalence continues to increase globally. According to the 2015 Global Burden of Disease (GBD) Study, there are 358 million people with asthma worldwide, an increase of $12.6 \%$ since 1990 (1). The Global Initiative for Asthma, Global Strategy for Asthma Management and Prevention (GINA) estimates that this number will increase to 400 million by 2025 (2).

In recent years, the rapid development of China's economy has accelerated urbanization resulting in major changes to people's living environment and lifestyle. The prevalence of asthma among children 14 years and younger in China's urban areas was $1.54 \%$ in 2000 (3) and $3.02 \%$ in 2010 (4), representing an increase of $96.1 \%$ in 10 years. The reported incidence of adult asthma varies due to differences in sampling methods used by different epidemiological surveys and the definitions of asthma. The results of the China Asthma and Risk Factors Epidemiologic Investigation Study (CARE Study) conducted in 8 provinces and cities of 7 administrative regions in China from 2010 to 2011 showed that the prevalence of asthma diagnosed by doctors in people over 14 years old was $1.24 \%$, and $26 \%$ of the patients with asthma were newly diagnosed (5). The China Pulmonary Health (CPH) Study, conducted from 2012 to 2015 in 10 provinces and cities of China, including 160 urban and rural sites, reported that the prevalence of asthma in persons aged 20 years and older was $4.2 \%$ (6).

Asthma severity is commonly assessed retrospectively based on the medications prescribed (2). Furthermore, changes in prescriptions at follow-up visits can be used to indicate worsening or improving asthma severity (7-9). To date, however, there are no large sample epidemiological data from China describing asthma severity at the time of diagnosis and the changes that occur during followup management. A previous cross-sectional study showed that of 2,034 persons diagnosed with asthma, 6\% were considered to have severe disease (10). In another crosssectional survey, $75 \%$ of 1,191 self-reported respondents were deemed to have mild asthma based on the medications they were prescribed (11).

This study used a large health care database to examine asthma severity at initial diagnosis, and to assess the changes in severity over the first year of management. These results may help to optimize asthma management and the allocation of medical resources.

We present the following article in accordance with the STROBE reporting checklist (12) (available at https://atm. amegroups.com/article/view/10.21037/atm-21-6470/rc).

\section{Methods}

\section{Data source}

The SuValue health information database is a structured, electronic hospital medical record system constructed based on ambulatory and hospitalization encounters. It contains de-identified patient data for all diseases from 2000 to March 2020. The database captures the full continuum of treatment information for more than 90 million patients from 207 hospitals in 18 provinces in China. The patient data can be longitudinally tracked, and includes demographic information, diagnoses, laboratory testing results, medications prescribed, procedures performed, and the costs for inpatient, outpatient, and emergency department care. The database was constructed and is available for research purposes with the full authorization of each participating hospital. Centennial Scientific Co., Ltd., has dedicated access to the SuValue database.

\section{Study design and population}

This was a retrospective study of newly diagnosed asthma patients. Eligible patients aged 14 years or more at the time of initial asthma diagnosis (ICD-10-CM: J45) between January 2001 and March 2019 were eligible for inclusion in this study. The diagnosis criteria referred to the Chinese Guidelines for Bronchial Asthma Prevention and Management. Patients must have had at least 2 non-asthma related clinic visits separated by at least 30 days over the 1 -year pre-index period to ensure the continuity of care (13). Patients were followed up for at least 12 months from the date of initial diagnosis (Figure 1) to reduce seasonal effect on asthma management.

All procedures performed in this study involving human participants were in accordance with the Declaration of Helsinki (as revised in 2013). Only existing data from which all personally identifiable information was removed by the database provider prior to receipt by the study team were used in this study. Thus, informed patient consent and institutional review board/ethics committee approval were not required as indicated by the local medical research policy for using electronic medical records.

\section{Assessment of asthma severity}

Patient demographic data and medications prescribed at the 


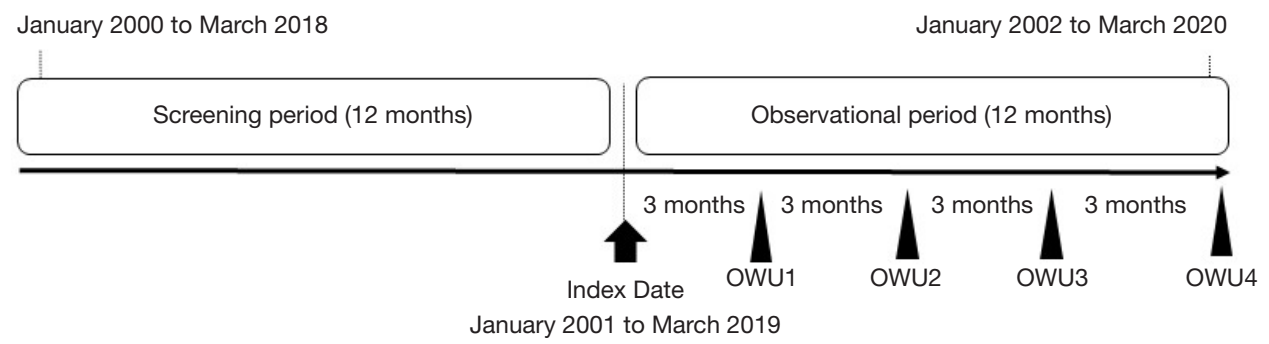

Figure 1 Study design. OWU: observation window unit, 1 OWU represents 3 months.

index date were collated from the medical records. A drugbased severity classification method was used according to the GINA 2021 stepwise treatment recommendations (2). Patients with asthma were categorized with severe, moderate, or mild asthma based on the intensity of the asthma therapy prescribed. In accordance with the Chinese Guidelines for Bronchial Asthma Prevention and Management 2020, patients with severe asthma were defined as those who were prescribed combination medium/ high-dose of inhalation corticosteroid and long-acting $\beta 2$-agonist (ICS/LABA), combination medium/high-dose ICS/LABA plus long-acting muscarinic antagonist (LAMA), or combination high-dose ICS plus leukotriene receptor antagonist (LTRA) or theophylline, with additional antiimmunoglobulin E monoclonal antibody, LAMA, or oral corticosteroid (OCS). Patients with moderate asthma were defined as those who were prescribed low-dose ICS/LABA, medium/high-dose ICS, or low-dose ICS plus LTRA or theophylline. Patients with mild asthma were defined as those not meeting the definition of either severe asthma or moderate asthma.

The proportion of patients with different asthma severities were recorded. Asthma severity classifications were summarized for consecutive observation window units (OWUs) which were defined as 3-month periods from the index date to the end of the 12-month follow-up. Patients were included in an $\mathrm{OWU}$ if they were seen within 1 month before or 1 month after the OWU. In situations where multiple medications were recorded for a given OWU, asthma severity classification was based on the last prescription (category and dosage) for the OWU.

\section{Statistical analysis}

Data are reported as mean \pm standard deviation or count (percentage). Subgroup analyses were performed using the Chi-square or the Fisher exact test. The unequal variance two-sample $t$-test was used for continuous data. All analyses were two-tailed, and a $\mathrm{P}$ value $<0.05$ was considered statistically significant.

\section{Results}

\section{Study population and patient characteristics}

A total of 7,654 patients were enrolled in this retrospective study. A flow diagram of the patient selection and exclusion process is shown in Figure 2. The mean age of the included patients was 52 years, and $54.18 \%$ were females. Patients greater than 60 years of age accounted for the largest proportion of patients $(37.66 \%$ ), while $26.39 \%$ of the study cohort were 14 to 40 years old. Patients from secondary and tertiary hospitals accounted for $73.62 \%$ and $26.38 \%$ of the study population, respectively (Table 1). Approximately $65 \%$ of patients were treated in internal medicine departments, $17 \%$ in emergency departments, and $11 \%$ in departments devoted to traditional Chinese medicine. However, the distribution of the departments where patients were treated varied with the level of care of the hospital. For example, in tertiary hospitals, patients were primarily treated in the respiratory medicine department $(70.74 \%)$ and internal medicine department (29.26\%), whereas, in secondary hospitals, patients were mainly treated in the internal medicine department $(59.40 \%)$, followed by the emergency department $(21.22 \%)$, and traditional Chinese medicine department (12.87\%) (Table 2).

\section{Initial severity and symptoms of asthma}

Of the 7,654 patients, $53.91 \%$ were not prescribed asthma control medications at the initial visit, suggesting that these patients had only mild intermittent symptoms $(<2$ times per month before diagnosis). However, $15.84 \%$ of patients received medications including OCS and ICS-LABA, suggesting a more severe condition with symptoms prior to 


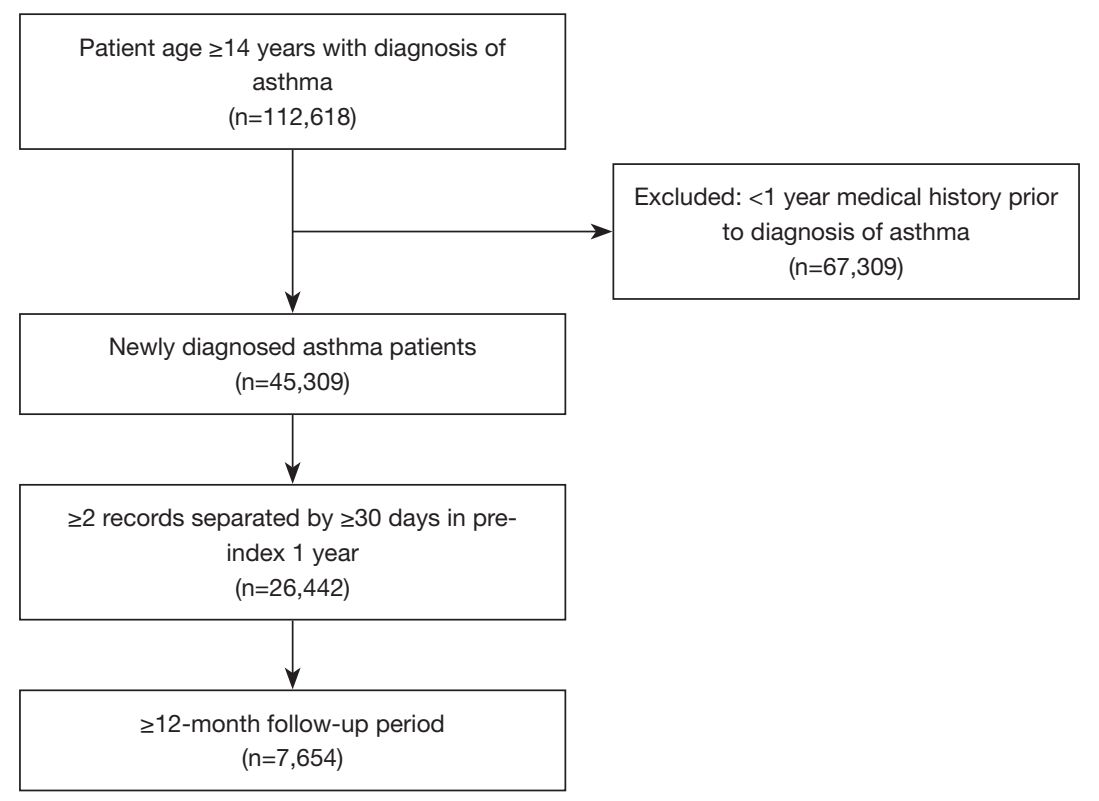

Figure 2 Flow diagram of patient selection.

Table 1 Patient characteristics $(\mathrm{N}=7,654)$

\begin{tabular}{lc}
\hline Characteristics & Value \\
\hline Gender, n (\%) & \\
Male & $3,507(45.82)$ \\
Female & $4,147(54.18)$ \\
Age, years, mean (SD) & $52(17.08)$ \\
$14-40$ & $2,020(26.39)$ \\
$41-60$ & $2,752(35.96)$ \\
$>60$ & $2,882(37.66)$ \\
Hospital, n (\%) & \\
Tertiary & $2,019(26.38)$ \\
Secondary & $5,635(73.62)$ \\
\hline
\end{tabular}

diagnosis (Table 3).

\section{Subsequent severity}

Over the year of observation, the overall proportion of patients with moderate and severe asthma decreased during the first 6 months and remained stable thereafter. The proportion of patients with severe asthma remained stable from 6 months. In the 4 observation windows (3, 6, 9, and 12 months), the mild asthma rates were $75.99 \%, 88.75 \%$,
$89.66 \%$, and $90.15 \%$, respectively, and the severe asthma rates were $5.33 \%, 2.56 \%, 2.55 \%$, and $2.7 \%$, respectively. At the end of the year, the proportion of patients with moderate and severe asthma was about $10 \%$, among which $2.7 \%$ had severe asthma (Figure 3).

During the 12-month follow-up, changes in asthma severity were noted in some patients. Patients with mild asthma tended to continue to have mild asthma in the following 3 months (>76.19\%). However, $92.85 \%$ and $75.1 \%$ of patients with mild and moderate asthma at 3 months, respectively, experienced maintenance and reduction in severity and at 12 months, these patients only had mild asthma. However, $1.26 \%$ of patients with mild asthma and $3.15 \%$ of patients with moderate asthma progressed to severe asthma by 12 months (Figure 4).

\section{Discussion}

To the best of our knowledge, this is the first study performed in China to investigate the initial asthma severity at diagnosis and the changes in severity over the first year of management. The results demonstrated that after 1 year, nearly $10 \%$ of patients had moderate or severe asthma. This study represents the actual clinical practice in China involving a heterogeneous adult and adolescent population treated in both tertiary and secondary hospitals with a wide regional coverage. The results of the study highlight 
Table 2 Distribution of initial asthma diagnosis

\begin{tabular}{|c|c|c|c|}
\hline Department & All, n (\%) & Tertiary, n (\%) & Secondary, n (\%) \\
\hline Internal medicine & 4,967 (64.89) & 1,620 (80.24) & 3,347 (59.40) \\
\hline Respiratory & 2,033 (26.56) & $1,146(56.76)$ & 887 (15.74) \\
\hline Non-respiratory & 2,934 (38.33) & $474(23.48)$ & $2,460(43.66)$ \\
\hline Chinese medicine & $863(11.28)$ & $138(6.84)$ & $725(12.87)$ \\
\hline Otolaryngology & $270(3.53)$ & $29(1.44)$ & 241 (4.28) \\
\hline Surgical & $112(1.46)$ & $53(2.63)$ & $59(1.05)$ \\
\hline Pediatric & $96(1.25)$ & $41(2.03)$ & $55(0.98)$ \\
\hline
\end{tabular}

Table 3 Treatment received at the initial asthma diagnosis

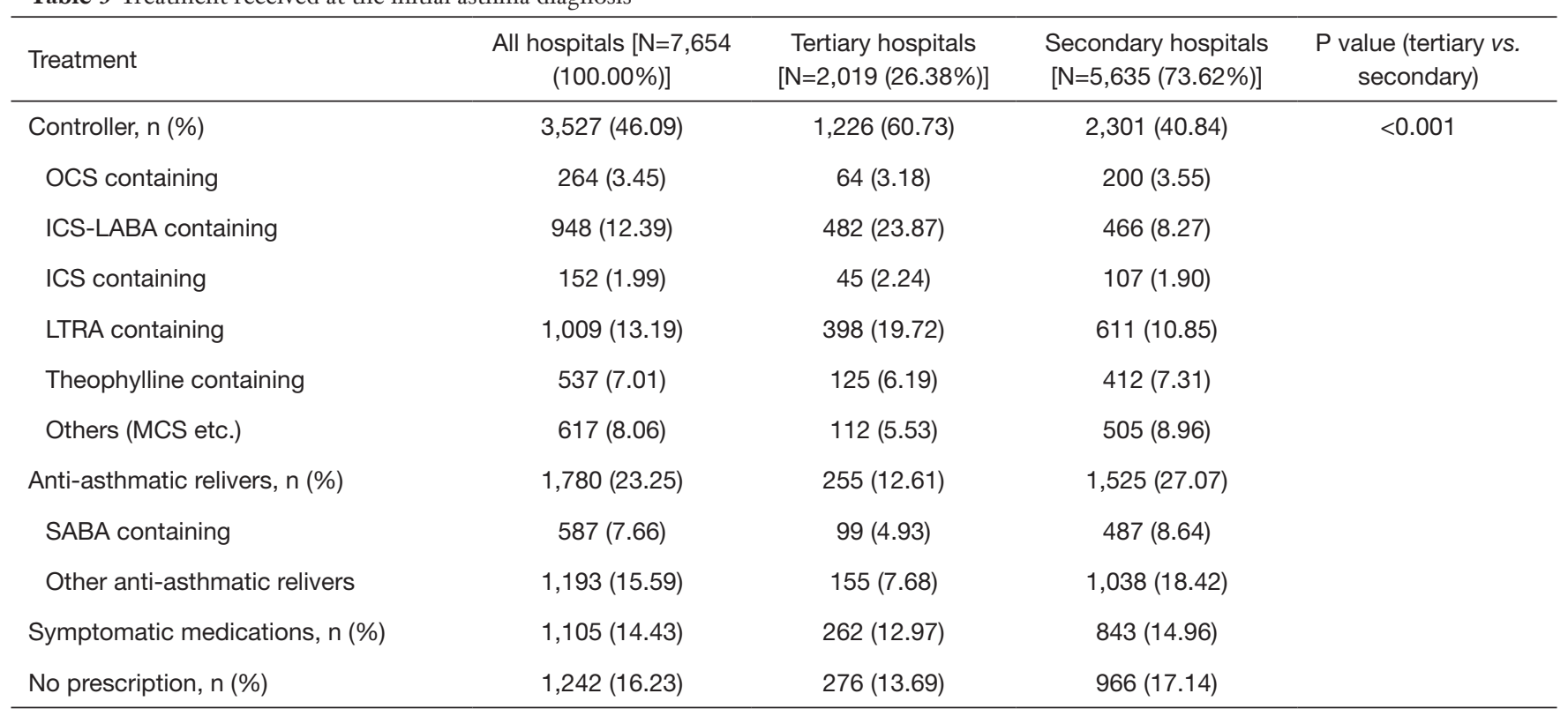

(I) Patients were categorized by the highest preferred treatment of each step, e.g., categorized as OCS if a combination of OCS and ICS-LABA was used, without overlapping counting in every category; (II) other anti-asthmatic relivers included SAMA, SABA/SAMA combination, short-acting xanthine derivatives, short course ( $\leq 7$ days) injective/oral/nebulized corticosteroids; (III) symptomatic medications included TCM, antitussive, mucolytics, cough-mucolytics combination, anti-histamine, and antibiotics. OCS, oral corticosteroid; ICS-LABA, inhaled corticosteroid and long-acting $\beta 2$-agonist combination; ICS, inhaled corticosteroid; LTRA, leukotriene receptor antagonist; MCS, mast cell stabilizers; SABA, short-acting 32 -agonist; TCM, traditional Chinese medicine.

the real-world management of asthma and complements previous data from cross-sectional population-based surveys.

Unlike other chronic diseases, there are no objective markers of underlying asthma disease severity. However, asthma severity can be assessed when a patient has been on controller treatment for several months. This study involving 7,654 newly diagnosed asthma patients, revealed that the prevalence of severe asthma was stable during a 1-year follow-up period. In our study cohort, $2.7 \%$ of patients presented with severe asthma at the 1-year followup, which is consistent with the $2.4 \%$ prevalence reported 


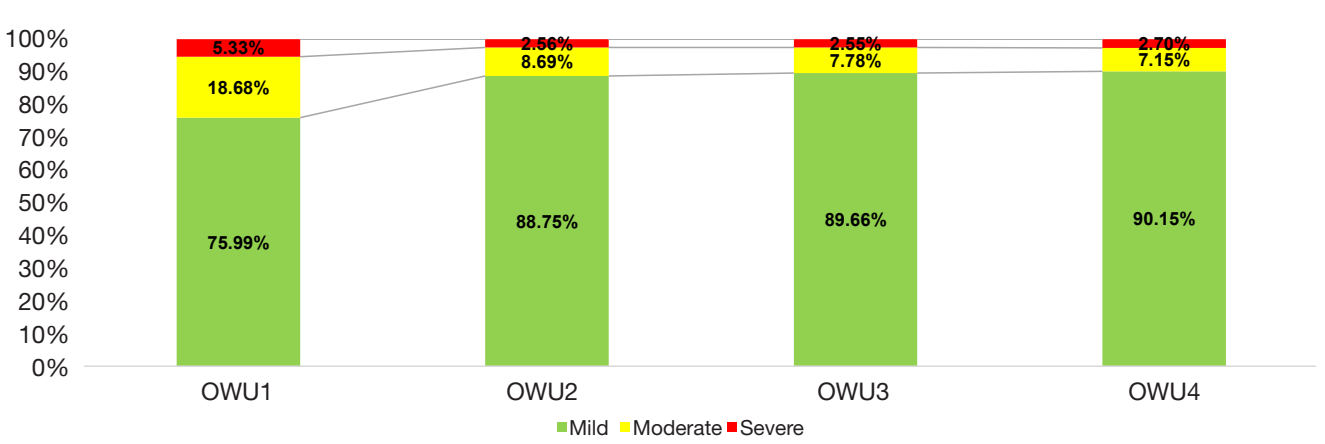

Figure 3 Distribution of asthma severity in each 3-month observation window unit. (I) OWU: observation window unit, 1 OWU represents 3 months; (II) asthma severity classification was based on the last prescription of controller medication; (III) a 1-month grace period before and after the OWU was permitted to account for patients' early or late visit.

in a retrospective study performed in Japan that used a nationwide health care claims database (14). However, the incidence of severe asthma was lower than the commonly cited prevalence of $5 \%$ to $10 \%(15,16)$. In a previous crosssectional study in China (10), the criteria for determining asthma severity were different to that used in our study (17). Data used in our study were obtained from continuous outpatient records, and there were no lapses of treatment or missing data with respect to any of our included patients.

Population-based studies and longitudinal studies in other countries have contributed significantly to our understanding of associations with initial disease severity and the changes in asthma over time. A cross-sectional survey of 3,509 children aged 5-14 years in Canada found that the prevalence of childhood asthma was significantly different between children living in urban locations and rural areas, and exposure to certain rural factors may aggravate the condition in asthmatic children (18). A population-based survey of over 12,000 adolescents in France showed that the prevalence of 'ever asthma' was higher among boys, whereas severe asthma was associated with early onset and females (19). The authors revealed that asthmatic adolescents required more health care in terms of medication use, consultations, and hospitalizations comparing to other adolescents, and this correlation was strongest among severe asthmatics. A Danish study followed asthmatics over a 30-year period and reported that the female gender, previous severe exacerbation(s), and older age at baseline were associated with uncontrolled asthma at follow-up and a blood-eosinophil count of $0.3 \times 10^{9} / \mathrm{L}$ or greater. Furthermore, being prescribed an ICS at baseline was associated with being prescribed a medium- or highdose ICS at follow-up (20). The authors concluded that asthma rarely remits in adults, especially in individuals with longer duration and more severe disease, and worse initial disease is associated with uncontrolled asthma at follow-up.

GINA guidelines provide specific recommendations for choice of pharmacotherapy, frequency of regular follow-up, and adjustment of therapy when needed. However, in many cases, the needs of an individual patient will differ from the medical goals. Poor adherence to therapy is common in patients with asthma and is often associated with increased use of health care resources, morbidity, and mortality, and this includes asthmatic patients in China $(21,22)$. A clear explanation of asthma and the disease severity by a physician can improve a patient's trust and confidence in the treatment regimen. Asthma severity is not a static feature and may change overtime, and in this current study, approximately $90 \%$ of patients presented with mild asthma at 12 months, even though a marked proportion of patients had moderate or severe asthma during the first 6 months. Importantly, our results demonstrated that, in general, patients diagnosed initially with mild asthma tend to continue to have mild asthma and progression to severe disease is not common. This finding can provide positive evidence to help to improve treatment confidence and adherence for better outcome.

The results revealed that a small group of patients developed severe asthma over the year, and some patients with severe asthma did not experience a reduction in severity over the year. As such, further study is warranted to examine the clinical features of newly diagnosed patients with severe asthma who do not experience a reduction in severity in order to target these patients for more intensive treatment and reduce the disease burden.

There are certain advantages in using data from a 


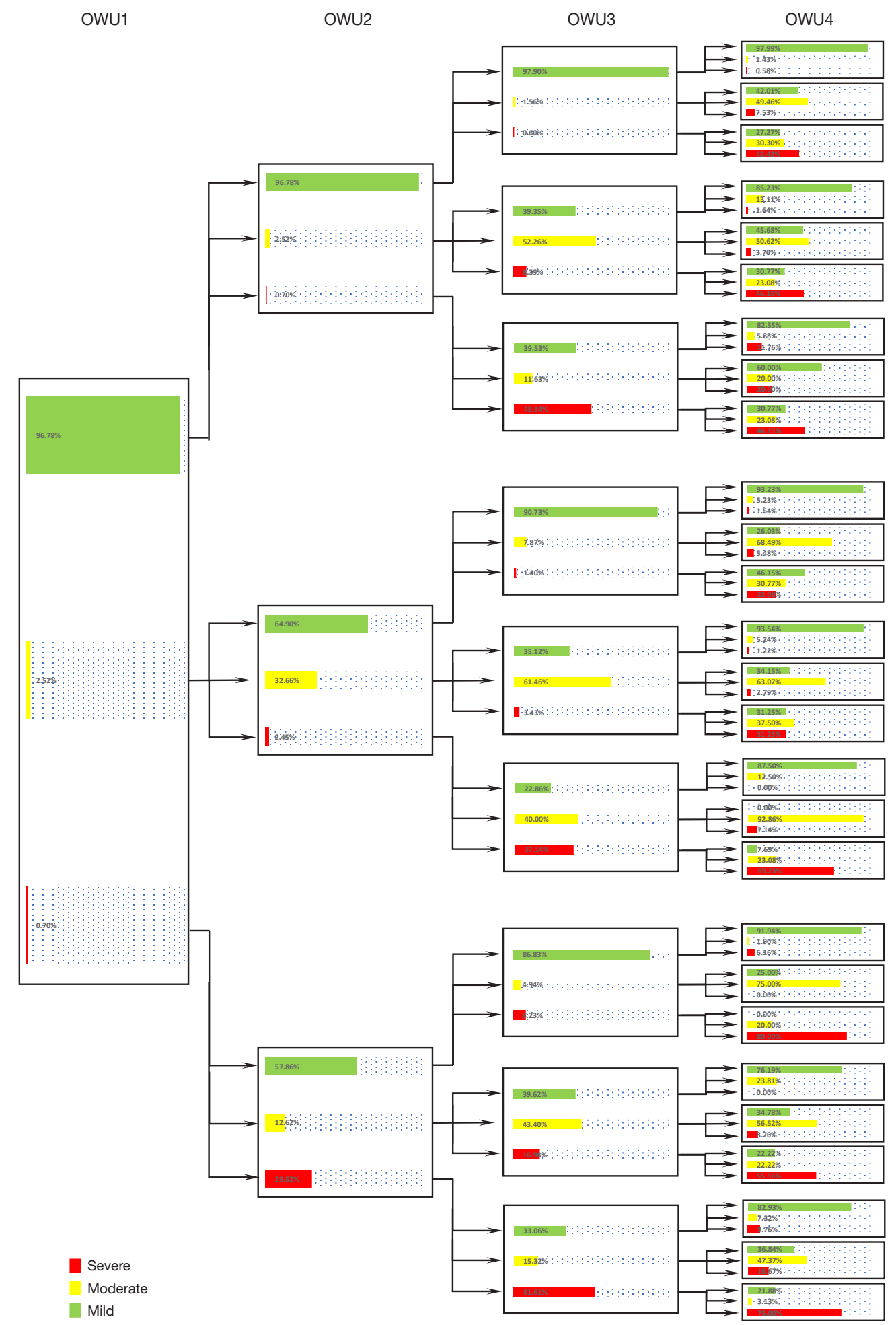

Figure 4 Changes in asthma severity in each 3-month observation window unit. OWU: observation window unit, 1 OWU represents 3 months. 
health information database, including the large sample size and the ability to identify all outpatient visits and hospitalizations. The latter allows follow-up of individual patients, which differs from most epidemiological studies that are cross-sectional and lack follow-up data. These advantages enabled the examination of real-world data regarding asthma management and complement the limitations of hospital-based studies. Furthermore, this study only included individuals who were actively engaged with their health care provider for ailments other than asthma before the index date. By doing so, the number of patients lost to follow-up for various reasons, such as loss of health insurance coverage, was reduced.

Conversely, there are some limitations inherent to studies that are based on data from medical records. Data retrieval is limited to the variables registered in the database, and medication use is based on medications prescribed which may not necessarily reflect patient compliance. This study classified asthma severity according to medications prescribed, not clinical data, and did not assess medication adherence. These shortcomings may have resulted in the misclassification of asthma severity in some patients. In addition, using asthma medications as a proxy for asthma severity relies on physician adherence to current practice guidelines (23). Deviation of asthma treatment from guidelines may result in inaccurate estimations of asthma severity and other potential biases (24).

\section{Conclusions}

This study demonstrated that during the first year after an initial diagnosis of asthma, patients with mild asthma tended to not progress further but remained stable with mild asthma over a 1-year follow-up period. The proportion of patients diagnosed with moderate and severe asthma also remained stable over 1 year. Further studies examining the clinical features of newly diagnosed patients with severe asthma who do not experience a reduction in severity should be conducted to facilitate intensive treatment and reduce the disease burden for these patients.

\section{Acknowledgments}

Funding: This study was supported by the Scientific and Technological Project of Guangdong Province (No. 2017B020226006) and the National Natural Science Foundation of China (Nos. 81970032, 81770033, and 81670026).

\section{Footnote}

Reporting Checklist: The authors have completed the STROBE reporting checklist. Available at https://atm. amegroups.com/article/view/10.21037/atm-21-6470/rc

Data Sharing Statement: Available at https://atm.amegroups. com/article/view/10.21037/atm-21-6470/dss

Conflicts of Interest: All authors have completed the ICMJE uniform disclosure form (available at https://atm. amegroups.com/article/view/10.21037/atm-21-6470/coif). YG, LL, and XH report that they are from Centennial Scientific Co., Ltd., The other authors have no conflicts of interest to declare.

Ethical Statement: The authors are accountable for all aspects of the work in ensuring that questions related to the accuracy or integrity of any part of the work are appropriately investigated and resolved. This is a retrospective analysis from a structured electronic hospital medical record system constructed and based on ambulatory and hospitalization encounters with de-identified patient level data and does not involve human participants, human data, nor human tissues.

Open Access Statement: This is an Open Access article distributed in accordance with the Creative Commons Attribution-NonCommercial-NoDerivs 4.0 International License (CC BY-NC-ND 4.0), which permits the noncommercial replication and distribution of the article with the strict proviso that no changes or edits are made and the original work is properly cited (including links to both the formal publication through the relevant DOI and the license). See: https://creativecommons.org/licenses/by-nc-nd/4.0/.

\section{References}

1. GBD 2015 Chronic Respiratory Disease Collaborators. Global, regional, and national deaths, prevalence, disability-adjusted life years, and years lived with disability for chronic obstructive pulmonary disease and asthma, 1990-2015: a systematic analysis for the Global Burden of Disease Study 2015. Lancet Respir Med 2017;5:691-706.

2. Global Initiative for Asthma, Global strategy for asthma management and prevention. Available online: https:// ginasthma.org/

3. Chen YZ; National Cooperation Group On Childhood 
Asthma. A nationwide survey in China on prevalence of asthma in urban children. Zhonghua Er Ke Za Zhi 2003;41:123-7.

4. National Cooperative Group on Childhood Asthma, Institute of Environmental Health and Related Product Safety, Chinese Center for Disease Control and Prevention, et al. Third nationwide survey of childhood asthma in urban areas of China. Zhonghua Er Ke Za Zhi 2013;51:729-35.

5. Lin J, Wang W, Chen P, et al. Prevalence and risk factors of asthma in mainland China: The CARE study. Respir Med 2018;137:48-54.

6. Huang K, Yang T, Xu J, et al. Prevalence, risk factors, and management of asthma in China: a national cross-sectional study. Lancet 2019;394:407-18.

7. Reddel HK, Taylor DR, Bateman ED, et al. An official American Thoracic Society/European Respiratory Society statement: asthma control and exacerbations: standardizing endpoints for clinical asthma trials and clinical practice. Am J Respir Crit Care Med 2009;180:59-99.

8. Taylor DR, Bateman ED, Boulet LP, et al. A new perspective on concepts of asthma severity and control. Eur Respir J 2008;32:545-54.

9. Chung KF, Wenzel SE, Brozek JL, et al. International ERS/ATS guidelines on definition, evaluation and treatment of severe asthma. Eur Respir J 2014;43:343-73.

10. Su N, Lin JT, Wang WY, et al. A cross-section study of severe asthma in eight provinces of China. Zhonghua Nei Ke Za Zhi 2016;55:917-21.

11. Ding B, DiBonaventura M, Karlsson N, et al. A crosssectional assessment of the prevalence and burden of mild asthma in urban China using the 2010, 2012, and 2013 China National Health and Wellness Surveys. J Asthma 2017;54:632-43.

12. von Elm E, Altman DG, Egger M, et al. The Strengthening the Reporting of Observational Studies in Epidemiology (STROBE) statement: guidelines for reporting observational studies. J Clin Epidemiol 2008;61:344-9.

13. Hernán MA, Robins JM. Using Big Data to Emulate a Target Trial When a Randomized Trial Is Not Available.

Cite this article as: $\mathrm{Hu} \mathrm{Z,} \mathrm{Xuan} \mathrm{J,} \mathrm{Zhao} \mathrm{H,} \mathrm{Dong} \mathrm{H}$, Yu C, Cai S, Gao Y, Li L, Hu X. Newly diagnosed asthma in China: initial severity and changes over a 1-year management period. Ann Transl Med 2022;10(2):75. doi: 10.21037/atm-21-6470
Am J Epidemiol 2016;183:758-64.

14. Sato K, Ohno T, Ishii T, et al. The Prevalence, Characteristics, and Patient Burden of Severe Asthma Determined by Using a Japan Health Care Claims Database. Clin Ther 2019;41:2239-51.

15. Bel EH, Sousa A, Fleming L, et al. Diagnosis and definition of severe refractory asthma: an international consensus statement from the Innovative Medicine Initiative (IMI). Thorax 2011;66:910-7.

16. O'Byrne PM, Naji N, Gauvreau GM. Severe asthma: future treatments. Clin Exp Allergy 2012;42:706-11.

17. Lin J. Chinese Thoracic Society Expert consensus on diagnosis and management of refractory asthma. Chinese Journal of Tuberculosis and Respiratory Diseases 2010;33:572-7.

18. Lawson JA, Rennie DC, Cockcroft DW, et al. Childhood asthma, asthma severity indicators, and related conditions along an urban-rural gradient: a cross-sectional study. BMC Pulm Med 2017;17:4.

19. Moreau D, Ledoux S, Choquet M, et al. Prevalence and severity of asthma in adolescents in France. Crosssectional and retrospective analyses of a large populationbased sample. Int J Tuberc Lung Dis 2000;4:639-48.

20. Tupper OD, Håkansson KEJ, Ulrik CS. Remission and Changes in Severity Over 30 Years in an Adult Asthma Cohort. J Allergy Clin Immunol Pract 202 1;9:15951603.e5.

21. Boulet LP, Vervloet D, Magar Y, et al. Adherence: the goal to control asthma. Clin Chest Med 2012;33:405-17.

22. Yuan Y, Li B, Huang M, et al. Fractional exhaled nitric oxide was not associated with the future risk of exacerbations in Chinese asthmatics: a non-interventional 1-year real-world study. J Thorac Dis 2019;11:2438-47.

23. Pont LG, van der Werf GT, Denig P, et al. Identifying general practice patients diagnosed with asthma and their exacerbation episodes from prescribing data. Eur J Clin Pharmacol 2002;57:819-25.

24. Hasford J, Uricher J, Tauscher M, et al. Persistence with asthma treatment is low in Germany especially for controller medication - a population based study of 483,051 patients. Allergy 2010;65:347-54. 